\title{
Staining of argininosuccinate lyase activity in polyacrylamide gel
}

\author{
Hwei-Jen Lee ${ }^{1}$, Shyh-Horng Chiou ${ }^{2}$ and Gu-Gang Chang ${ }^{1}$ \\ ${ }^{1}$ Department of Biochemistry, National Defence Medical Center and ${ }^{2}$ Instituie of Biochemical Sciences, \\ National Taiwan University and Institute of Biological Chemistry, Academia Sinica, Taipei, \\ Taiwan, R.O.C.
}

(Received 8 October 1991)

(Accepted 9 December 1991)

\section{Summary}

A procedure for the direct staining of argininosuccinate lyase activity in polyacrylamide gel is described. The method was based on coupling one of the enzymatic products fumarate with fumarase and malic enzyme catalyzed reactions. Fumarate was first converted to L-malate by fumarase. Malic enzyme then catalyzed the oxidative decarboxylation of L-malate to give $\mathrm{CO}_{2}$ and pyruvate with concomitant reduction of NADP ${ }^{+}$to NADPH. Finally the reducing power of NADPH was coupled to phenazine methosulfate and in turn to nitroblue tetrazolium yielding a deeply colored insoluble formazan which may be quantitized or semiquantitized by densitometer.

Key words: Argininosuccinate lyase; $\delta$-Crystallin; Gel electrophoresis; Enzyme staining

\section{Introduction}

Argininosuccinate lyase (L-argininosuccinate arginine-lyase, EC 4.3.2.1) is a key enzyme in the urea cycle catalyzing the reversible cleavage of argininosuccinate to give $\mathrm{L}$-arginine and fumarate. Serum argininosuccinate lyase activity was shown to be a sensitive index for parachymal liver disease [1,2], whereas erythrocyte argininosuccinate lyase activity was used in the diagnosis of a recessive inherited disease argininosuccinic aciduria due to the deficiency of argininosuccinate lyase [3].

Abbreviations: PAGE, polyacrylamide gel electrophoresis; SDS, sodium dodecyl sulfate. Correspondence address: H.-J. Lee, Dept. of Biochemistry, National Defence Medical Center, P.O. Box 90048, Taipei, Taiwan, R.O.C. 
Recently some lens structural proteins were found to have similar amino acid sequence with the metabolic enzymes [4,5]. Among them $\delta$-crystallin isolated from duck eye-balls was found to be homologous with argininosuccinate lyase [6]. These unexpected findings have opened avenues to study the evolution of lens crystallins with enzymatic functions and their possible biological significance.

We are involved in a program of characterization of crystallins with enzymatic activities from the vertebrate lenses in order to shed some insight on the biological significance of the presence of enzymatic activity in the structural lens proteins. In our crystallin isolation procedure, we have obtained several $\delta$-crystallins from duck [7]. All of them possessed argininosuccinate lyase activity [8]. On the other hand, $\delta$-crystallin from chicken lens possessed very little enzymatic activity. Furthermore, the apparent homogeneous protein preparation as judged by SDS-PAGE revealed multiple bands when electrophoresis was performed under native condition. It is thus extremely important to develop a direct activity staining method for the argininosuccinate lyase activity in gels.

Nelson et al. [9] have developed an activity stain method for argininosuccinate lyase after starch gel electrophoresis. The arginine released was coupled to arginase, urease and glutamate dehydrogenase. The activity of argininosuccinate lyase was detected by the defluorescence of NADPH and located by an UV lamp. However, the method has not been critically evaluated.

In this paper, we describe an alternative procedure for the activity stain of argininosuccinate lyase. The sequential reaction steps we adapted were:

$$
\begin{aligned}
& \text { Argininosuccinate } \stackrel{\text { argininosuccinate lyase }}{\rightleftarrows} \text { Arginine + Fumarate } \\
& \text { Fumarate }+\mathrm{H}_{2} \mathrm{O} \stackrel{\text { fumarase }}{\rightleftarrows} \text { L-Malate }
\end{aligned}
$$

L-Malate $+\mathrm{NADP}+\stackrel{\text { malic enzyme }}{\rightleftarrows} \mathrm{CO}_{2}+$ Pyruvate $+\mathrm{NADPH}+\mathrm{H}^{+}$

The reducing power of NADPH was then coupled to phenazine methosulfate, an artificial electron acceptor, and finally to a tetrazolium dye which upon reduction polymerized and formed a highly-colored formazan precipitate at the site showing enzymatic activity. For a review and comprehensive survey on the enzyme localization in gels, see Ref. 10.

\section{Materials and Methods}

\section{Materials}

Argininosuccinate lyase (EC 4.3.2.1) (Type IV, from bovine liver, $0.5 \mathrm{U} / \mathrm{mg}$ ), fumarase (EC 4.2.1.2) (from porcine heart, affinity purified, $360 \mathrm{U} / \mathrm{mg}$ ), argininosuccinate, $\mathrm{NADP}^{+}$, phenazine methosulfate, and nitroblue tctrazolium were purchased from Sigma, St. Louis, MO. PhastGels were from Pharmacia-LKB Biotechnology. Other chemicals used were of highest grade available from Sigma or E. Merck (Germany). 
The barium salt of argininosuccinic acid from Sigma was converted to the potassium salt according to the method of Ratner [11]. The concentration of argininosuccinate was checked by the method of Kuchel et al. [12].

Malic enzyme (EC 1.1.1.40) from pigeon liver was purified to homogeneity according to our previously described procedure [13].

$\delta$-Crystallins were purified from duck eye-ball by a one-step TSK-DEAE-650(M) anion exchange chromatography, as described previously [7]. Three peaks of $\delta$-crystallin were obtained by $0.05-0.5 \mathrm{M}$ ammonium bicarbonate linear gradient elution. All of them were shown to have $M_{\mathrm{r}}$ of 200000 composed of four subunits with $M_{\mathrm{r}} 50000$. Peak 2 (with $0.29 \mathrm{U} / \mathrm{mg}$ argininosuccinate lyase activity) was used in this study. Protein concentration of the purified $\delta$-crystallin was determined spectrophotometrically at $280 \mathrm{~nm}$ using an absorption coefficient of 5.5 for a $1 \%$ $(w / v)$ protein solution.

\section{Assay of argininosuccinate lyase}

Assay of argininosuccinate lyase activity was performed at $25^{\circ} \mathrm{C}$. The formation of fumarate was monitored continuously at $240 \mathrm{~nm}$ in a Varian DMS-100 spectrophotometer. The reaction mixture contained $50 \mathrm{mM}$ Tris- $\mathrm{HCl}$ buffer (pH 7.5), $0.2 \mathrm{mM}$ argininosuccinate and an appropriate amount of protein in a total volume of $1 \mathrm{ml}$. One unit of enzyme activity was defined as an initial rate of $1 \mu \mathrm{mol}$ of fumarate released per minute under the assay conditions. A molar absorption coefficient of $2.44 \cdot 10^{3} \mathrm{M}^{-1} \mathrm{~cm}^{-1}$ for fumarate was used for calculation [14].

\section{Polyacrylamide gel electrophoresis under native conditions}

Electrophoresis was performed in duplicate with a Pharmacia-LKB PhastSystem in PhastGel homogeneous $12.5 \%$ mini-slab gel and PhastGel Native Buffer Strip (containing $0.88 \mathrm{M}$ L-alanine, $0.25 \mathrm{M}$ Tris- $\mathrm{HCl}, \mathrm{pH}$ 8.8). The samples were subjected to electrophoresis at $400 \mathrm{~mA}$ and $15^{\circ} \mathrm{C}$ for $220 \mathrm{~V} \mathrm{~h}$. After electrophoresis, one of the gel was subjected to protein stain with Coomassie brilliant blue R350 and the other for enzyme activity stain.

Activity stain solution consisted of $0.1 \mathrm{M}$ Tris-HCl buffer (pH 7.7), $1 \mathrm{mM}$ argininosuccinate, $7 \mathrm{U} / \mathrm{ml}$ fumarase, $2.8 \mathrm{mM} \mathrm{MnCl}{ }_{2}, 0.38 \mathrm{mM} \mathrm{NADP}{ }^{+}, 0.1 \mathrm{U} / \mathrm{ml}$ malic enzyme, $0.4 \mathrm{mg} / \mathrm{ml}$ nitroblue tetrazolium, and $0.07 \mathrm{mg} / \mathrm{ml}$ phenazine methosulfate. The stain solution was prepared just before use. In some cases, $\mathbf{0 . 1 \%}$ Tween-80 was included in the stain solution, if so, the solution should be shaken well to dissolve Tween-80. Degas or flushing the solution with $\mathrm{N}_{2}$ to remove $\mathrm{O}_{2}$ is preferred.

Enzymatic activity stain was performed by immersing the gel into $5-10 \mathrm{ml}$ stain solution. The color appeared after about $30-\mathrm{min}$ incubation at $37^{\circ} \mathrm{C}$ and deepened after several hours. Sometimes the gel was allowed to stay in stain solution overnight at room temperature for full color development. The reaction was stopped by replacing the stain solution with $10 \%$ ethanol-5\% acetic acid fixing solution. This stain method was sensitive to light and oxygen and should be performed in dark under closed chamber. The degased stain solution was sealed with parafilm for this purpose. 
The stained gels were scanned with a densitometer (Densitron Pan-FV, Jookoo, Japan) at $570 \mathrm{~nm}$, using a slit of $0.2 \mathrm{~mm}$ and full scale of $1 \mathrm{~A}$. The scan rate was 40 $\mathrm{mm} / \mathrm{min}$.

\section{Results}

Duck $\delta$-crystallin has high argininosuccinate lyase activity as measured by the conventional $A_{240}$ UV absorption method [6]. Since purified $\delta$-crystallin was readily available to us, we chose this protein as our major material while used commercially available argininosuccinate lyase as a control. Argininosuccinate lyase activity was stained violet-blue by the the method we described in this paper. The color appeared after $30 \mathrm{~min}$ at $37^{\circ} \mathrm{C}$ and deepened after overnight incubation. The correlation of incubation time with color yield is presented in Fig. 1. The densitometric scan of the gels is shown in Fig. 1B. Linear correlation between the color yield and incubation time was obtained up to $240 \mathrm{~min}$ (Fig. 1C). Fig. 2 shows the activity stain of duck $\delta$-crystallin at various protein concentrations. The lowest detection limit of the present method was about $0.2 \mu \mathrm{g}$ (Fig. 2A, gel 2) corresponding to $58 \cdot 10^{-6} \mathrm{U}$, representing an absorbance change of only 0.000142 per min if the enzyme activity was assayed by the conventional kinetic method. The densitometric scan of the gels is shown in Fig. 2B. Linear correlation between the color yield and $\delta$-crystallin applied was obtained when the protein was less than $1 \mu \mathrm{g}$ $\left(2.9 \cdot 10^{-4} \mathrm{U}\right)$ (Fig. 2C). Fig. 2 also demonstrates that other unrelated proteins $\left(M_{\mathrm{r}}\right.$ markers) were not stained by the stain solution (Fig. 2A, right panel, lane 7).

A commercially available argininosuccinate lyase was also stained positive by this method (Fig. 3, lane h). We also performed various controls to rule out any possibility of artifacts. All components in the stain solution, including $\delta$-crystallin, argininosuccinate, fumarase, malic enzyme, $\mathrm{Mn}^{2+}$, and NADP ${ }^{+}$, were found to be essential. Removing any component from the stain solution caused the staining not to work. The result of removing substrate from the stain solution was shown in Fig. 3 (lane e). To ensure this negative control, this gel was stained overnight. This negative result clearly ruled out the possibility of the so called "nothing-dehydrogenase" artifact noticed for other dehydrogenase stain (for review see Ref. 10). Replacing $\mathrm{NADP}^{+}$with $\mathrm{NAD}^{+}$still did not stain the activity, indicating that malic enzyme was specifically involved. Inclusion of $45 \mathrm{mM}$ L-citrulline, which specifically inhibited the argininosuccinate lyase activity of bovine liver [15] and of duck $\delta$-crystallin [8], in the stain solution decreased the color yield (Fig. 3, lane f). The present stain method was also applicable to crude duck lens extract (Fig. 3, lane c). We also tried to include Tween-80 in the stain solution as used by Worsfold et al. [16]. However, no significant improvement was obtained in sensitivity or resolution. The effect of Tween-80 on trapping formazan in solution seemed not to be applicable in gel.

The effect of substrate concentration on the color yield was also investigated. The densitometric scan of the gels is shown in Fig. 4. 
(A)
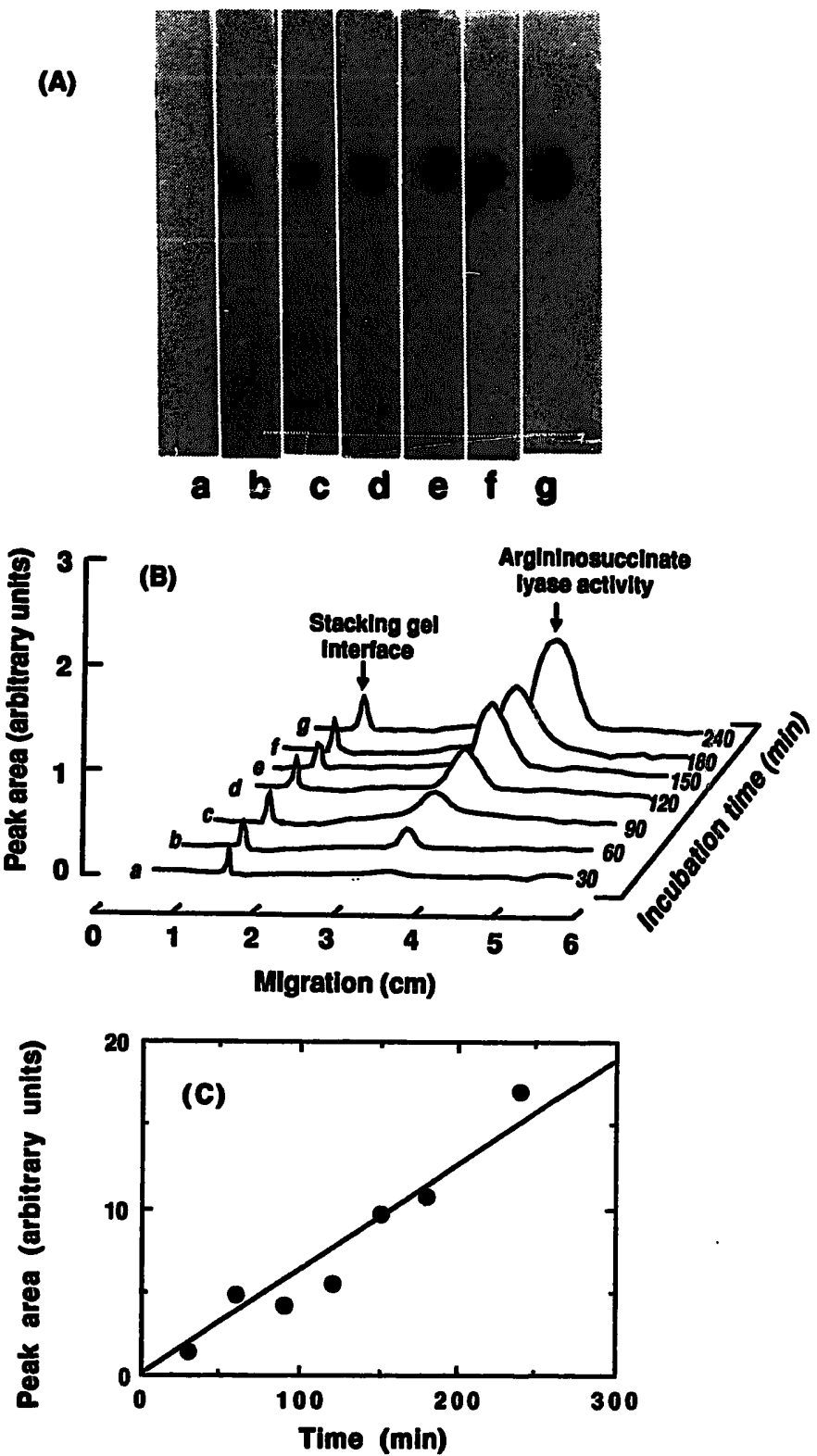

Fig. 1. Effect of incubation time on the color development of argininosuccinate lyase activity stain of duck $\delta$-crystallin in polyacrylamide gel. (A) Electrophoresis of duck $\delta$-crystallin $\left(4.2 \mu \mathrm{g}, 1.2 \cdot 10^{-3}\right.$ unit) was performed with Pharmacia pre-cast $12.5 \%$ PhastGel at $400 \mathrm{mV}$ for $220 \mathrm{~V}$ h. The staining time at $37^{\circ} \mathrm{C}$ was: (a) $30 \mathrm{~min}$, (b) $50 \mathrm{~min}$, (c) $90 \mathrm{~min}$, (d) $120 \mathrm{~min}$, (e) $150 \mathrm{~min}$, (f) $180 \mathrm{~min}$, (g) $240 \mathrm{~min}$. (B) Densitometric scanning of the argininosuccinate lyase activity of duck $\delta$-crystallin in gels. The gels in (A) were subjected to gel scan with slit of $0.2 \mathrm{~mm}$. (C) Relationship between incubation time and peak areas.

\section{Discussion}

We have described a direct localization and semi-quantitative staining method for the argininosuccinate lyase activity in polyacrylamide gel. The procedure is 
(A)

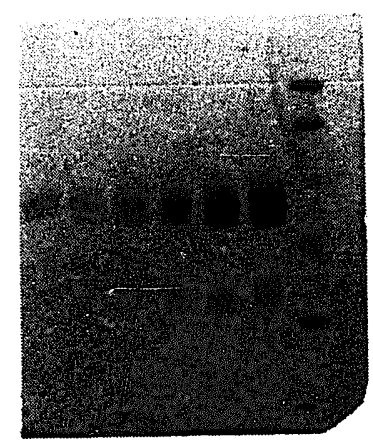

1234567

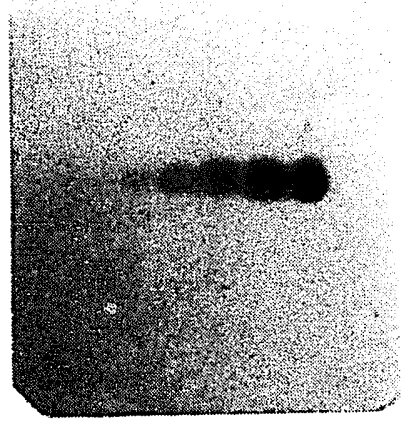

1234567
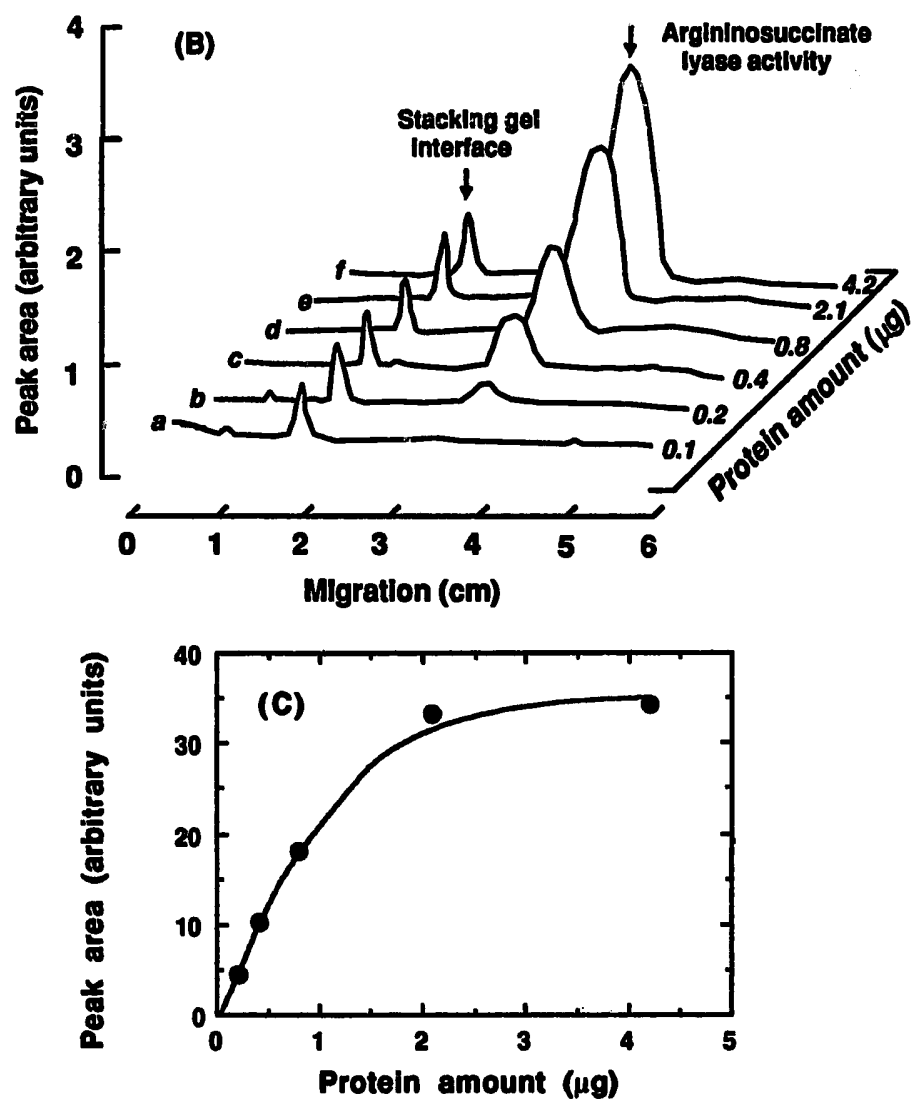

Fig. 2. Polyacrylamide gel electrophoresis of duck $\delta$-crystallin under native conditions. (A) Electrophoresis was performed as described in Fig. 1 with various amount of duck $\delta$-crystallin. Left panel: protein stain with Coomassie brilliant blue R-350. Right panel: argininosuccinate lyase activity stain. (1-6) duck $\delta$-crystallin: (1) $0.11 \mu \mathrm{g}$, (2) $0.21 \mu \mathrm{g}$, (3) $0.42 \mu \mathrm{g}$, (4) $0.84 \mu \mathrm{g}$, (5) $2.1 \mu \mathrm{g}$, (6) $4.2 \mu \mathrm{g}$. (7) $M_{\mathrm{r}}$ marker (from bottom to top: albumin, $M_{\mathrm{r}}$ 67000; lactate dehydrogenase, $M_{\mathrm{r}} 140000$; catalase, $M_{\mathrm{r}}$ 232000; ferritin, $M_{\mathrm{r}}$ 440000; thyroglobulin, $M_{\mathrm{r}}$ 669000). (B) Densitometric scanning of the argininosuccinate lyase activity of duck $\delta$-crystalline in gel. The gel in (A) (right panel) was subjected to gel scanning with slit of $0.2 \mathrm{~mm}$. (C) Relationship between $\delta$-crystallin amount and peak areas.

simple. Only a single incubation with all components is involved. Economy of the reagents is an advantage by using the mini PhastGels. The positive results presented in this paper confirm the kinetic assay data that duck $\delta$-crystallin indeed 


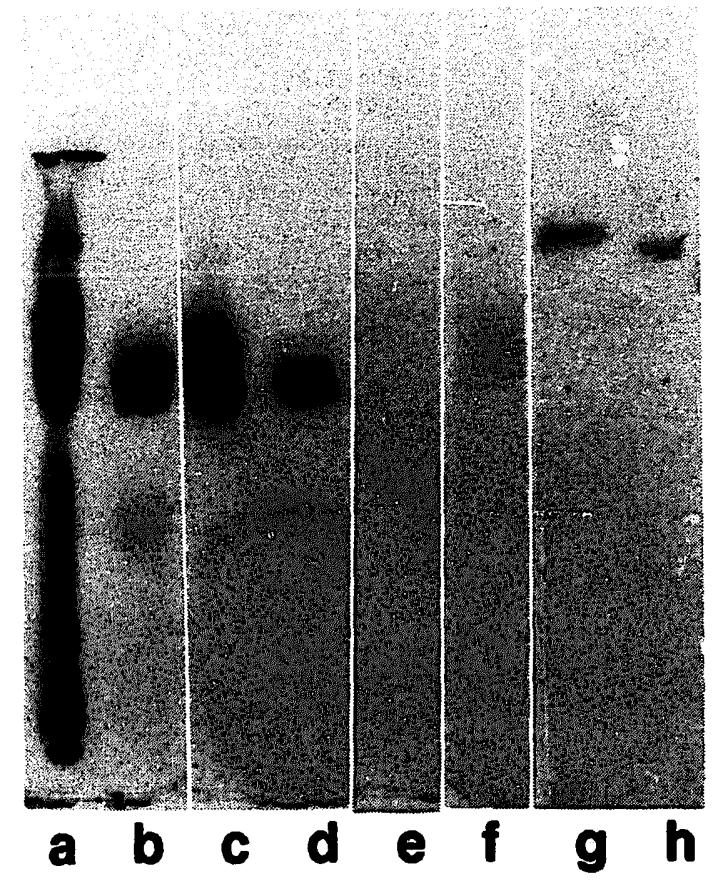

Fig. 3. Staining of argininosuccinate lyase activity under various conditions. Lanes $a$ and $b$, stained for protein; lanes $c-h$, stained for enzyme activity; lanes a and c, crude duck lens extract (56 $\mu \mathrm{g})$; lanes b, $\mathrm{d}-\mathrm{g}$, purified duck $\delta$-crystallin $\left(4.2 \mu \mathrm{g}, 1.2 \cdot 10^{-3}\right.$ unit); lane $\mathrm{h}$, commercial bovine liver argininosuccinate lyase $\left(1.9 \mu \mathrm{g}, 0.95 \cdot 10^{-3} \mathrm{U}\right)$. Gels $\mathrm{c}, \mathrm{d}, \mathrm{g}, \mathrm{h}$, stained for enzyme activity with complete stain solution at $37^{\circ} \mathrm{C}$ for $\mathbf{2 ~} \mathrm{h}$ as described in Materials and Methods; gel e, stained overnight with complete system minus argininosuccinate; gel f, stained with complete system plus $45 \mathrm{mM}$ L-citrulline. For gels a-f, electrophoresis conditions were the same as in Fig. 1. Gels $g$ and $h$ were electrophoresed with a 20\% PhastGel and run for $280 \mathrm{~V}$ h.

possesses genuine argininosuccinate lyase activity. In the development of an enzymatic method for the assay of serum argininosuccinate lyase, Miura et al. [17] have demonstrated that the argininosuccinate lyase-mediated reaction is the ratelimiting step when coupling with fumarase, malic enzyme and diaphorase. Argininosuccinate lyase activity was usually assayed in phosphate buffer [12,14,18]. However, this buffer was not suitable in the coupling system described in this paper since manganese ions precipitate in high concentration of phosphate solution.

All reagents used except pigeon liver malic enzyme were commercially available. We used pigeon liver malic enzyme simply because it was readily available to us. We believe that the commercially available chicken liver malic enzyme will perform just as well. Malic enzyme might be substituted by malate dehydrogenase (EC 1.1.1.37) but without obvious advantage. In the latter case, $\mathrm{NADP}^{+}$should be replaced by $\mathrm{NAD}^{+}$, and $\mathrm{Mn}^{2+}$ is not necessary. However, the $\mathrm{pH}$ optimum for malate dehydrogenase was around 9.5 , which was significantly higher than those for the argininosuccinate lyase and fumarase. Two stages may be required if using malate dehydrogenase, as suggested by Sherwin and Natelson [19] in the kinetic coupling assay. 

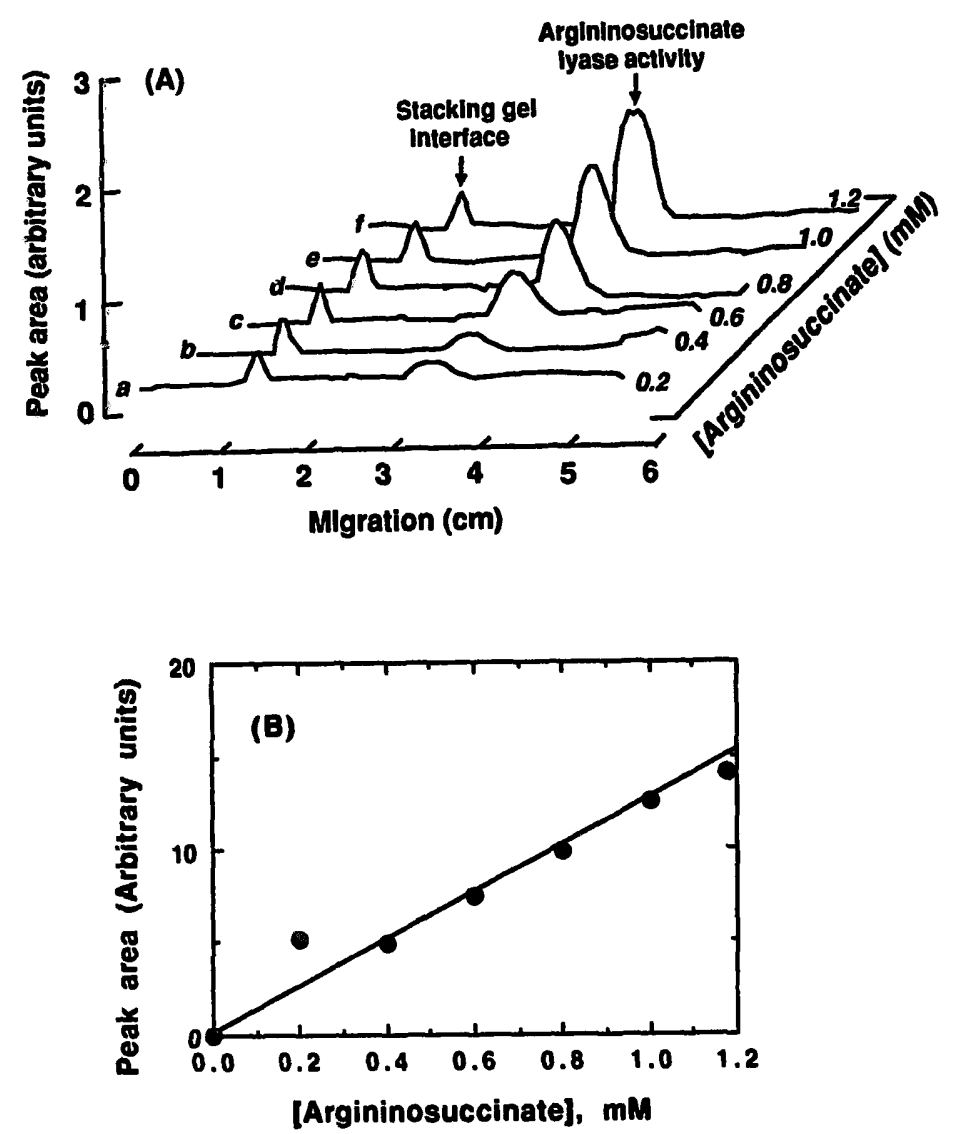

Fig. 4. Argininosuccinate lyase activity of duck $\delta$-crystallin in gels stained with various argininosuccinate concentrations. (A) Densitometric scanning of the gels. (B) Relationship between argininosuccinate concentration and peak area.

Several controls were tested to avoid erroneous interpretation. All components in the stain solution were found to be essential. L-Citrulline, a specific inhibitor for argininosuccinate lyase, was shown to decrease the color yield. The dependence of color yield on substrate concentration clearly ruled out the possibility of artificial staining.

The sensitivity of the present method was demonstrated to be much better than the conventional kinetic assay which followed the formation of fumarate at 240 $\mathrm{nm}$. Our result suggested that the gel stain method is about 10 -fold more sensitive than the conventional assay even using the most sensitive spectrophotometer. However, it may be less sensitive than other improved assay methods using coupling enzymes [17] or radioactive substrate [20,21]. We have demonstrated the correlation between $\delta$-crystallin amount applied (Fig. 2) or incubation time (Fig. 1) with the color yield. However, one should be very careful in interpreting densitometric data from enzymatic stains. We found that the response of color yield to substrate concentration was less sensitive than the conventional kinetic assay. Therefore, we suggest the use of the gel stain results only for qualitative or semiquantitative purposes. 


\section{Simplified description of the method}

Staining of argininosuccinate lyase activity in polyacrylamide gel was achieved by coupling one of the enzymatic products fumarate with fumarase and malic enzyme catalyzed reactions. Location of enzymatic activity was visualized as a deeply colored band which may be quantitized or semiquantitized by densitometer.

\section{Acknowledgment}

This work was supported in part by the National Science Council (NSC 81-0412-B016-33), R.O.C.

\section{References}

1 Campanini, R.Z., Tapia, R.A., Sarnat, W. and Natelson, S. (1970) Evaluation of serum argininosuccinate lyase (ASAL) concentrations as an index to parenchymal liver disease. Clin. Chem. 16, 44-53.

2 Takahara, K. and Natelson, S. (1967) Argininosuccinic acid lyase in human erythrocytes and plasma in health and disease. Am. J. Clin. Pathol. 47, 693-703.

3 Walsor, M. (1983) Argininosuccinic aciduria. In: Stanbury, J.B., Wyngaarden, J.B., Fredrickson, D.S., Goldstein, J.I. and Brown, M.S. (eds.), The Metabolic Basis of Inherited Diseases, McGrawHill, New York, pp. 402-438.

4 Wistow, G.J. and Piatigorsky, J. (1987) Recruitment of enzymes as lens structural proteins. Science 236, 1554-1556.

5 Wistow, G.J., Mulders, J.W.M. and de Jong, W.W. (1987) The enzyme lactate dehydrogenase as a structural protein in avian and crocodilian lenses. Nature 326, 622-624.

6 Piatigorsky, J., O'Brien, W.E., Norman, B.L., Kalumuck, K., Wistow, G.J., Borras, T., Nickerson, J.M. and Wawrousek, E.F. (1988) Gene sharing by $\delta$-crystallin and argininosuccinate lyase. Proc. Natl. Acad. Sci. USA 85, 3479-3483.

7 Chiou, S.-H., Lee, H.-J. and Chang, G.-G. (1990) Kinetic analysis of duck $\epsilon$-crystallin, a lens structural protein with lactate dehydrogenase activity. Biochem. J. 267, 51-58.

8 Lee, H.J., Chiou, S.H. and Chang, G.G. (1992) Biochemical characterization and kinetic analysis of duck $\delta$-crystallin with endogenous argininosuccinate lyase activity. Biochem. J. (in press).

9 Nelson, R.L., Povey, S., Hopkinson, D.A. and Harris, H. (1977) Detection after electrophoresis of enzymes involved in ammonia metabolism using L-glutamate dehydrogenase as a linking enzyme. Biochem. Genet. 15, 1023-1035.

10 Heeb, M.J. and Gabriel, O. (1984) Enzyme localization in gels. In: Jakoby, W.B. (Ed.), Methods in Enzymology, Vol. 104, Academic Press, Orlando, FL, pp. 416-439.

11 Ratner, S. (1957) Preparation and determination of argininosuccinic acid. In: Colowick, S.P. and Kaplan, N.O. (Eds.), Methods in Enzymology, Vol. 3, Academic Press, New York, pp. 643-647.

12 Kuchel, P.W., Nichol, L.W. and Jeffrey, P.D. (1975) Physicochemical and kinetic properties of beef liver argininosuccinase. Studies in the presence and absence of arginase. Biochim. Biophys. Acta 397, 478-488.

13 Chang, J.T. and Chang, G.G. (1982) Purification of pigeon liver malic enzyme by affinity chromatography. Anal. Biochem. 121, 366-369.

14 Ratner, S. (1970) Argininosuccinase (steer liver). In: Taber, H. and Taber, C.W. (Eds.), Methods in Enzymology, Vol. 17A, Academic Press, New York, pp. 304-309.

15 Raushel, F.M. and Nygaard, R. (1983) Kinetic mechanism of bovine liver argininosuccinate lyase. Arch. Biochem. Biophys. 221, 143-147. 
16 Worsfold, M., Marshall, M.J. and Ellis, E.B. (1977) Enzyme detection using phenazine methosulphate and tetrazolium salts: interference by oxygen. Anal. Biochem. 79, 152-156.

17 Miura, T., Kashiwamura, M. and Kimura, M. (1987) An enzymatic method for the assay of serum argininosuccinate lyase. Anal. Biochem. 164, 482-487.

18 O'Brien, W.E. and Barr, R.H. (1981) Argininosuccinate lyase: purification and characterization from human liver. Biochemistry 20, 2056-2060.

19 Sherwin, J.E. and Natelson, S. (1975) Serum and erythrocyte argininosuccinate lyase assay by NADH fluorescence generated from formed fumarate. Clin. Chem. 21, 230-234.

20 Zidwick, M.J. and Rogers, P. (1982) The determination by radiochemical assay of argininosuccinase produced in an Escherichia coli system in vitro. Biochem. J. 207, 529-533.

21 Ratner, S. and Kimiko, M.-M. (1980) A new radiochemical assay for argininosuccinase with purified $\left[{ }^{14}\right.$ C]argininosuccinate. Anal. Biochem. 106, 134-147. 